\title{
Prevalence of coeliac disease in children and adolescents with type 1 diabetes mellitus in a tertiary hospital in South Africa
} \author{
J C van Dyk, ${ }^{1}$ MMed (Paed) \\ ${ }^{1}$ Department of Paediatric Endocrinology, University of Pretoria, South Africa \\ ${ }^{2}$ University of Pretoria Diabetes Research Centre, South Africa \\ ${ }^{3}$ Department of Paediatric Gastroenterology, University of Pretoria, South Africa \\ ${ }^{4}$ Department of Endocrinology, University of Pretoria, South Africa
}

M Karsas, ${ }^{1,2}$ FC Paed Cert (SA); A J Terblanche, ${ }^{3}$ FC Paed Cert (SA); T Kemp, ${ }^{1,2,4}$ FC Paed Cert (SA);

Corresponding author: M Karsas (mkarsas@hotmail.com)

\begin{abstract}
Background. International literature has shown the prevalence of coeliac disease $(\mathrm{CD})$ in children and adolescents with diabetes to range from $1-10 \%$. Prevalence rates in African countries are limited or unknown.

Objective. The objective was to describe the prevalence of CD in all children and adolescents with type 1 diabetes mellitus presenting to the paediatric and adult diabetic clinic at Steve Biko Academic Hospital, Pretoria, South Africa.

Method. A retrospective review of the files of all children and adolescents in the paediatric and adult diabetic clinic with type 1 diabetes mellitus between August 2016 and June 2019 was conducted. Children requiring screening and/or intestinal biopsies were also prospectively included during this period. The setting of this study was Steve Biko Academic Hospital, a tertiary referral centre, in Pretoria, South Africa. Coeliac screening included anti-deaminated gliadin antibodies and anti-tissue transglutaminase antibodies (both IgA and $\mathrm{IgG}$ ). All biopsies were obtained by a paediatric gastroenterologist or an experienced paediatric surgeon.

Results. A total of 184 files were screened; 132 met inclusion criteria but only 108 patients in total had coeliac screening. Positive antibody screening for $\mathrm{CD}$ was found in 11 out of 108 patients (10.2\%). Nine of the 11 serology-positive patients had biopsies performed. Out of the nine biopsies, two (22.2\%) were positive for CD based on the Marsh-Oberhuber classification.

Conclusion. This study found a prevalence of serology-positive CD in our local population of South African children with type 1 diabetes mellitus of $10.2 \%$, while the prevalence of biopsy-confirmed CD was found to be $1.9 \%$.
\end{abstract}

S Afr J Child Health 2021;15(4):212-217. https://doi.org/10.7196/SAJCH.2021.v15i4.1841

Coeliac disease $(\mathrm{CD})$ is a chronic inflammatory autoimmune intestinal disease. It develops as a result of an interplay among immunological, genetic and environmental factors. ${ }^{[1]}$ Genetically predisposed individuals who ingest gluten develop an inflammatory enteropathy, characterised by intra-epithelial lymphocyte proliferation, crypt hyperplasia as well as complete or partial small intestinal villous atrophy with subsequent malabsorption. ${ }^{[2,3]}$

International literature has shown the prevalence of $\mathrm{CD}$ in children and adolescents with diabetes to range from 1 - 10\%, with an incidence of about 8 per 1000 patients per year. ${ }^{[4,5]}$ Studies conducted in developed countries show a prevalence rate of CD in children with type 1 diabetes mellitus of $5 \%$ in Turkey and $10 \%$ in Danish children. In a paediatric diabetes clinic in London, serology-confirmed CD prevalence was $6.2 \%$, while $4.4 \%$ was biopsy confirmed. ${ }^{[6-8]}$ The largest study performed, which included 52721 youth with diabetes mellitus across Europe (Germany, Austria, England and Wales), the USA and Australia showed an overall prevalence rate of biopsy-confirmed $\mathrm{CD}$ of $3.5 \%$, with prevalence rates ranging from $1.9 \%$ in the USA to $7.7 \%$ in Australia. ${ }^{[5,9]}$

In developing countries, far fewer data are available on the prevalence of $\mathrm{CD}$, particularly in African countries. A study conducted in a paediatric endocrinology clinic in Western India showed a prevalence (based on serology) of $15.49 \%$, while biopsyconfirmed CD prevalence was $7.04 \% \cdot{ }^{[10]}$ The prevalence rate in Brazil was reported to be $3.1 \%$; a prevalence of $5.5 \%$ was reported in Omani children, and $10.4 \%$ non-biopsied confirmed CD was found in Saudi Arabian children. ${ }^{[11-13]}$ Four studies were conducted on the prevalence of $\mathrm{CD}$ in Iranian children with type 1 diabetes mellitus; the first, conducted in 2004, showed a prevalence of $3.4 \%$ while the second report was a review based on three studies conducted and was published in 2014, showing a combined study prevalence of 5.66\%. In African countries, research in Egypt showed a prevalence of $6.4 \%, 16.4 \%$ in Algeria and 2.3\% biopsy-proven CD in Tunisia. ${ }^{[14-16]}$ In South Africa, a study conducted in KwaZuluNatal reported a prevalence of positive coeliac serology in type 1 diabetic children of $44.5 \%$. However, only $16 \%$ of these patients underwent biopsy, of whom three $(0.06 \%)$ had biopsy-confirmed CD. The small number of biopsies performed was attributed to lack of a qualified paediatric gastroenterologist; this challenge resulted in a significant limitation to the study. ${ }^{[17]}$ A later study of adult type 1 diabetic patients in the same region, which included patients who were diagnosed in childhood (with earliest onset of diagnosis of 10.3 years) showed a prevalence of $32.2 \%$ for serology-positive CD, while the prevalence of biopsy-confirmed CD was $2.5 \%$ (similar to Western countries). ${ }^{[18]}$

The diagnosis of $\mathrm{CD}$ is based on a combination of serology testing, small-intestinal biopsy and response to a gluten-free diet. ${ }^{\left[{ }^{3]}\right.}$ Confirmation of the diagnosis of $\mathrm{CD}$ can be made by demonstrating subtotal villus atrophy (as outlined in the Marsh Classification) on small-bowel biopsy. ${ }^{[4,5,19]}$ Despite the high prevalence of CD in 
children with type 1 diabetes mellitus, there has been much debate regarding routine screening in this population. ${ }^{[7]}$

Studies on the prevalence of CD in children with type 1 diabetes mellitus are limited, especially in developing countries. Current guidelines for screening children with $C D$ are based on international guidelines, as there is a lack of regional and national data on the prevalence of $\mathrm{CD}$ in South African (SA) children with type 1 diabetes mellitus. Knowledge regarding the prevalence of $\mathrm{CD}$ in our setting will assist in the application of international guidelines in our resource-limited environment based on local prevalence rates.

\section{Method}

The objective of the present study was to investigate the prevalence of $\mathrm{CD}$ in all children and adolescents with type 1 diabetes mellitus presenting to the paediatric diabetic clinic at Steve Biko Academic Hospital, a tertiary referral centre, in Pretoria, SA.

The study design was a retrospective review of the files of all children and adolescents in the paediatric diabetic clinic with type 1 diabetes mellitus between August 2016 and January 2019. Children requiring screening and/or intestinal biopsies were also prospectively included during this period. Exclusion criteria for this study were all children with type 2 diabetes mellitus, neonatal diabetes mellitus, maturity-onset diabetes of the young, secondary diabetes mellitus, and adults (over the age of 18 years).

Clinical information and signs or symptoms of $\mathrm{CD}$ were reviewed from the charts retrospectively. The following serology was recorded, namely tissue transglutaminase antibodies (tTG-A) IgA and IgG antibodies, antibodies against deaminated forms of gliadin peptides (antiDPG) IgA and IgG antibodies and total IgA, which was routinely done to exclude IgA deficiency. For testing, a Thermo Fischer Scientific (South Africa) kit was used; both tTG-A and anti-DPG IgA and $\operatorname{IgG}$ were tested via fluorimetric enzyme immunoassays. All above-mentioned serological tests were deemed positive on the kit if $\geq 10 \mathrm{U} / \mathrm{mL}$ or equivocal if $\geq 7 \mathrm{U} / \mathrm{mL}$ but $<10 \mathrm{U} / \mathrm{mL}$. Endomysial antibodies (EMA) are unfortunately not performed by our laboratory. $\mathrm{HbA1C}$ and diabetes autoantibodies were also recorded. Patients who had positive coeliac serology had intestinal biopsies taken via gastroscopy. Laboratory and histology results relied on the experience of laboratory technicians and pathologists for accuracy of results. All biopsies in our setting were obtained by a paediatric gastroenterologist (in seven patients) or a paediatric surgeon with experience in gastroscopies (in two patients) according to coeliac screening protocols with at least $4-6$ biopsies from the distal duodenum and 2 - 4 biopsies from the duodenal bulb. All serological tests and biopsies were obtained while children were on gluten-containing diets. Duodenal biopsies were examined under light microscopy using the modified Marsh classification. ${ }^{[19]}$ The diagnosis of $\mathrm{CD}$ was based on the European Society for Paediatric Gastroenterology, Hepatology and Nutrition (ESPGHAN) and the International Society for Pediatric and Adolescent Diabetes (ISPAD) guidelines. ${ }^{[5,20]}$ ESPGHAN and ISPAD guidelines state that a small-bowel biopsy demonstrating subtotal villus atrophy (as outlined in the Marsh classification), while the patient is on a gluten-containing diet, is required to confirm the diagnosis of $\mathrm{CD}$ once elevated antibodies are detected. ${ }^{[5,19,20]}$ However, for clearly symptomatic children with tTG-A titres $\geq 10$ times upper limit of normal (x ULN), CD may be diagnosed without a duodenal biopsy if the patient has a positive HLA DQ2 or DQ8 haplotype and the EMA IgA is also positive. ${ }^{[5,20]}$ In the latter, ESPGHAN requires only additional EMA IgA positivity; HLA determination and symptoms are not obligatory criteria for the diagnosis of CD. ${ }^{[20]}$

Data were collected on an Exel spreadsheet (Microsoft Corp., USA) and analysed using STATA (v15.1; STATACorp., USA). Categorical variables were assessed using the Fisher's exact test and Pearson's $\chi^{2}$ test, and continuous variables were assessed using the Wilcoxon's rank sum test. A $p$-value $<0.05$ was considered statistically significant.

The information gathered was treated confidentially and no patients' names were recorded. Informed consent was obtained from the parents/guardians and informed assent from all children between 8 and 17.9 years of age for all children added prospectively. The study obtained permission and approval from Steve Biko Academic Hospital. Ethical approval was obtained from the Research Ethics Department of the University of Pretoria (695/2018) and the National Health Research Database.

The potential benefits of this study included knowledge regarding the prevalence of CD in children and adolescents with type 1 diabetes mellitus in our setting, which will aid us in drawing up guidelines for CD screening in our population. There were no potential harms or known conflicts of interest associated with this study.

\section{Results}

The results of this study are depicted in the flow diagram in Fig. 1, showing 10.2\% of the patients to have serology-positive $\mathrm{CD}$, while only $22.2 \%$ of these patients had biopsyconfirmed CD.

\section{Patient demographics}

Of the 132 patients who met inclusion criteria for the study, the majority were

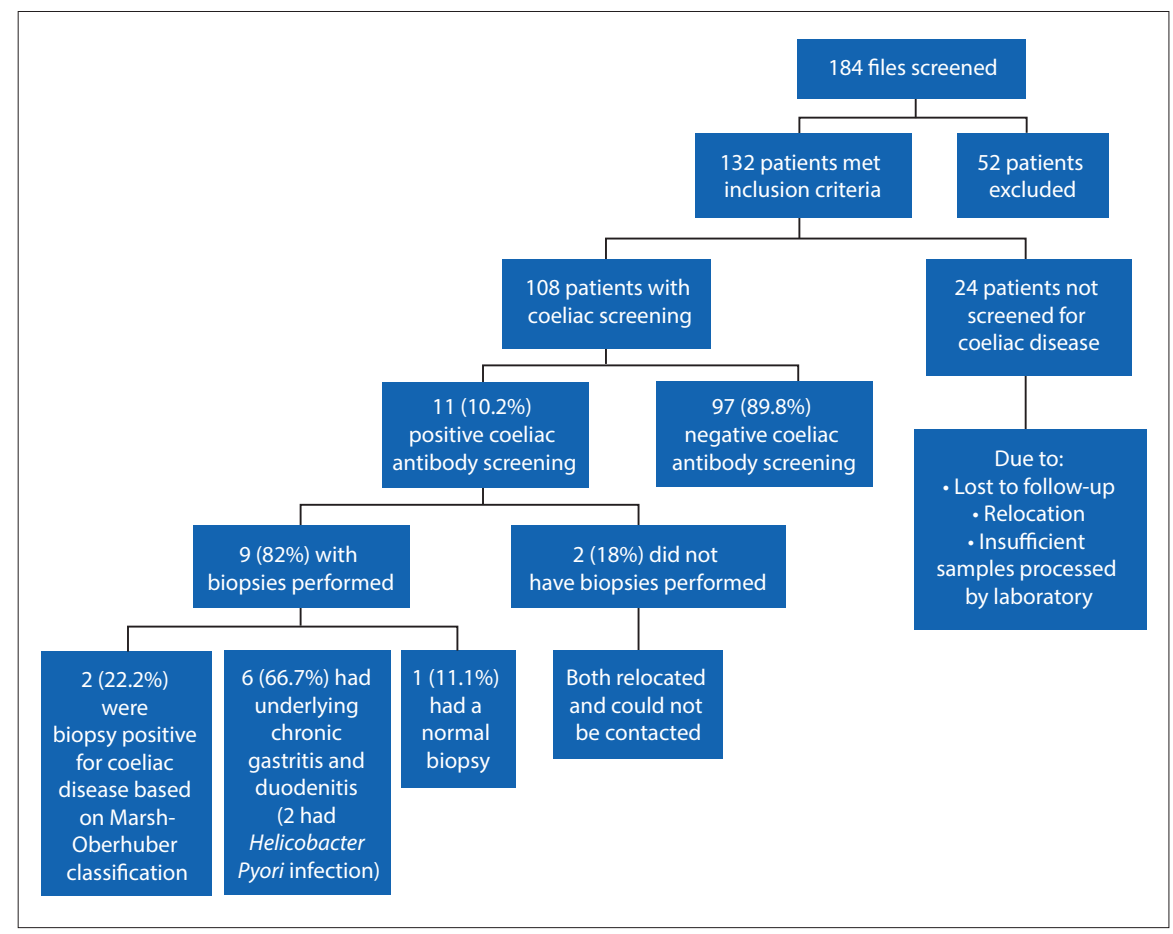

Fig. 1. Flow diagram of patient screening, coeliac serology and histology results. 
female $(54 \%)$, of whom most $(65 \%)$ were black, while $24 \%$ were white, $6 \%$ coloured, $4 \%$ Indian and $1 \%$ Asian. Ethnicity of the patient compared with biopsy-confirmed CD was not significant ( $p$-value 0.0629 ). Biopsy positivity specifically in the female gender v. male was statistically significant ( $p$-value 0.007 ) (Table 1).

The mean age of patients was 11.7 years (range 1.2 - 18 years), with a median of 12.3 years. The mean duration of diabetes was 4.4 years (range 0.1 - 17 years), with a median of 3.5 years. The mean $\mathrm{HbA1C}$ was $11.1 \%$ (range $5.1-20.2$ ), with a median HbAlC of also $11 \%$. The age of the patient compared with biopsy-confirmed CD was not significant ( $p$-value 0.0874 ). The duration of diabetes compared with biopsy-confirmed $\mathrm{CD}$ or serology positivity was also not significant ( $p$-value 0.9333 and 0.6116 , respectively) (Table 1 ).

\section{Coeliac serology}

Coeliac serology was deemed positive if tTG-A and/or anti-DPG were positive or equivocal. All $11(100 \%)$ patients had positive antiDPG (IgA and/or IgG) and five (45\%) also had positive tTG-A (IgA and/or IgG). None (0\%) of patients screened had an IgA deficiency. The greater the positivity of the coeliac serology antibodies $(\geq 10$ $x$ ULN) and the combination of positive anti-DPG plus tTG-A significantly increased the chances of a positive biopsy ( $p$-value 0.003 and 0.001 , respectively) (Table 2). Eight (72.7\%) of the 11 serology-positive patients were black, two $(18.2 \%)$ were coloured (mixed race) and one (9\%) was white. The mean age of the serologypositive patients was 8.5 years (range 3.3 - 13.9 years). There was a predominance of serology positivity in females of $82 \%$ (nine patients). Thirty-three (25\%) of the 132 patients screened had signs or symptoms of $\mathrm{CD}$; however, only three $(9 \%)$ of the symptomatic patients had positive coeliac antibodies and none ( $0 \%)$ had biopsyconfirmed CD. Of the patients who had positive coeliac screens, 73\% (8 patients) were asymptomatic.

Table 1. Summary of $p$-value results

\begin{tabular}{ll}
\hline Outcome & $p$-value \\
\hline Ethnicity compared to biopsy-confirmed CD & 0.0629 \\
CD biopsy positivity compared to gender & 0.0070 \\
Age compared to biopsy-confirmed CD & 0.0874 \\
Duration of diabetes compared to biopsy-confirmed CD & 0.9333 \\
Duration of diabetes compared to serology positivity & 0.6116 \\
CD = coeliac disease. &
\end{tabular}

Interestingly, as seen in Fig. 1, only one $(11.1 \%)$ out of the nine gastrointestinal biopsies taken was found to be completely normal; this patient was a 5-year-old black girl. Two were confirmed to have CD: a 4-year-old white girl and a 7-year-old black girl, both with CD grade 3a Modified Marsh-Oberhuber classification. The mean age of biopsy-confirmed CD was six years, with a female predominance of $100 \%$. Both patients with confirmed CD had coeliac serology testing with both antibodies (tTG-A and anti-DPG) $\geq 10 \mathrm{x}$ ULN and both were asymptomatic. The other six (66.7\%) biopsies were abnormal, showing a picture of chronic gastritis and chronic duodenitis. Two of these six abnormal biopsies also cultured Helicobacter pylori infection for which the patients received eradication; one patient also had a co-existing Giardia infection. The demographics of these six patients were quite varied and included two boys and four girls, with an age range of $3-12$ years, and were of black and coloured descent. All patients with abnormal biopsies not confirming CD had antibodies $<10 \times$ ULN.

Statistically, the diabetes-associated antibody positivity rate compared to biopsy-confirmed CD was not significant ( $p$-value 0.276 ); out of a total 86 patients who had confirmed positive diabetesassociated antibodies, only one (1.2\%) had biopsy-confirmed CD. The presence of signs and symptoms of CD compared with biopsyconfirmed CD was also not significant ( $p$-value 0.514$)$; 103 patients had documented signs and symptoms, $74(71.8 \%)$ were asymptomatic for $\mathrm{CD}$ and only two (1.9\%) patients had biopsy-confirmed CD and both were asymptomatic.

\section{Discussion}

As seen in the results of this study, the prevalence rate in our population of diabetic children and adolescents in Pretoria, South Africa, of serology-positive CD was $10.2 \%$. This is much lower than the reported prevalence of $44.5 \%$ and $32.2 \%$ in paediatric and adult patients, respectively in Durban, South Africa. ${ }^{[17,18]}$ This may occur as a result of different antibody testing. Serology was positive based on either tTG-A or EMA, while in the adult study patients' serology was deemed positive if any of the three antibodies were positive (tTG-A, EMA, anti-gliadin antibodies [AGA]). ${ }^{[17,18]}$ EMA and tTG-A both have a specificity and sensitivity $>90 \%$ in symptomatic individuals (Table 3). ${ }^{[3-5,21]}$ When used as screening tests, however, their positive predictive value is lower, in the range of $70-83 \% .{ }^{[21]}$ The population studied was investigated with a panel of tests, rather than screened with a single antibody test as recommended in the ESPGHAN and ISPAD guidelines. Our laboratory, unfortunately, does not test for

Table 2. Breakdown of positive/equivocal coeliac serology results and correlation with biopsy positivity

\begin{tabular}{|c|c|c|c|c|c|c|c|c|c|c|c|}
\hline Patient & 1 & 2 & 3 & 4 & 5 & 6 & 7 & 8 & 9 & 10 & 11 \\
\hline \multicolumn{12}{|l|}{$\begin{array}{l}\text { tTG-A } \\
(\mathrm{U} / \mathrm{ml})\end{array}$} \\
\hline $\operatorname{IgA}$ & $\begin{array}{l}\mathbf{P} \\
200.0\end{array}$ & $\begin{array}{l}\mathbf{P} \\
288.0\end{array}$ & $\begin{array}{l}\mathbf{E} \\
8.2\end{array}$ & $\begin{array}{l}\mathrm{N} \\
2.3\end{array}$ & $\begin{array}{l}\mathrm{N} \\
0.5\end{array}$ & $\begin{array}{l}\mathrm{N} \\
0.8\end{array}$ & $\begin{array}{l}\mathrm{N} \\
0.4\end{array}$ & $\begin{array}{l}\mathbf{E} \\
8.8\end{array}$ & $\begin{array}{l}\mathrm{N} \\
6.7\end{array}$ & $\begin{array}{l}\mathrm{N} \\
0.3\end{array}$ & $\begin{array}{l}\mathrm{N} \\
1.4\end{array}$ \\
\hline IgG & $\begin{array}{l}\mathbf{P} \\
12.0\end{array}$ & $\begin{array}{l}\mathbf{P} \\
46\end{array}$ & $\begin{array}{l}\mathrm{N} \\
2.2\end{array}$ & $\begin{array}{l}\mathbf{P} \\
60\end{array}$ & $\begin{array}{l}\mathrm{N} \\
0.5\end{array}$ & $\begin{array}{l}\mathrm{N} \\
0.0\end{array}$ & $\begin{array}{l}\mathrm{N} \\
0.4\end{array}$ & $\begin{array}{l}\mathbf{E} \\
7.2\end{array}$ & $\begin{array}{l}\mathrm{N} \\
1.0\end{array}$ & $\begin{array}{l}\mathrm{N} \\
0.0\end{array}$ & $\begin{array}{l}\mathrm{N} \\
1.2\end{array}$ \\
\hline $\begin{array}{l}\text { Anti-DPG } \\
(\mathrm{U} / \mathrm{ml})\end{array}$ & & & & & & & & & & & \\
\hline $\operatorname{Ig} \mathrm{A}$ & $\begin{array}{l}\mathbf{P} \\
96\end{array}$ & $\begin{array}{l}\mathbf{P} \\
302\end{array}$ & $\begin{array}{l}\mathbf{P} \\
10.1\end{array}$ & $\begin{array}{l}\mathrm{N} \\
0.1\end{array}$ & $\begin{array}{l}\mathbf{P} \\
12.0\end{array}$ & $\begin{array}{l}\mathbf{E} \\
9.1\end{array}$ & $\begin{array}{l}\mathbf{E} \\
7.8\end{array}$ & $\begin{array}{l}\mathrm{N} \\
2.4\end{array}$ & $\begin{array}{l}\mathrm{N} \\
3.7\end{array}$ & $\begin{array}{l}\mathbf{P} \\
29.0\end{array}$ & $\begin{array}{l}\mathbf{P} \\
13.0\end{array}$ \\
\hline IgG & $\begin{array}{l}\mathbf{P} \\
164\end{array}$ & $\begin{array}{l}\mathbf{P} \\
815\end{array}$ & $\begin{array}{l}\mathbf{E} \\
7.1\end{array}$ & $\begin{array}{l}\mathbf{P} \\
18\end{array}$ & $\begin{array}{l}\mathrm{N} \\
1.4\end{array}$ & $\begin{array}{l}\mathrm{N} \\
0.1\end{array}$ & $\begin{array}{l}\mathrm{N} \\
1.3\end{array}$ & $\begin{array}{l}\mathbf{P} \\
16.0\end{array}$ & $\begin{array}{l}\mathbf{P} \\
11.0\end{array}$ & $\begin{array}{l}\mathrm{N} \\
1.1\end{array}$ & $\begin{array}{l}\mathrm{N} \\
0.5\end{array}$ \\
\hline Coeliac disease on biopsy & $\mathbf{P}$ & $\mathbf{P}$ & $\mathrm{N}$ & $\mathrm{N}$ & $\mathrm{N}$ & $\mathrm{N}$ & $\mathrm{N}$ & $\mathrm{N}$ & $\mathrm{N}$ & ND & ND \\
\hline
\end{tabular}


Table 3. Sensitivity and specificity of different serological tests used in the diagnosis of CD (adapted from Pelkowski and Viera ${ }^{[3]}$ )

\begin{tabular}{lllll}
\hline Serological test & Sensitivity (\%) & Specificity (\%) & Positive likelihood ratio & Negative likelihood ratio \\
\hline IgG DPG & 80 & 98 & 40 & 0.20 \\
IgA DPG & 88 & 95 & 17.6 & 0.13 \\
IgG tTG-A & 40 & 95 & 8 & 0.63 \\
IgA tTG-A & $95-98$ & $94-95$ & 17.5 & 0.04 \\
IgG EMA & 40 & 95 & 8 & 0.63 \\
IgA EMA & $>90$ & $>95$ & $>18$ & $<0.11$ \\
IgG AGA & 80 & 80 & 4 & 0.25 \\
IgA AGA & $80-90$ & $85-95$ & 8.5 & 0.17 \\
CD = coeliac disease. & & & &
\end{tabular}

EMA; however, we included anti-DPG (which is more specific than AGA) as part of our serological testing. It is important to note that children younger than two years old in particular lack EMA and tTG antibodies and therefore serology testing in children younger than even five years old is thought to be less reliable and requires additional investigation. ${ }^{[1,3]}$ The specificity of coeliac serology testing in black SA patients is also unknown; this may have an influence on the results obtained.

The prevalence of definite biopsy-confirmed CD was found to be $1.9 \%$ in the present study. This is in keeping with the international literature that has shown the prevalence of $\mathrm{CD}$ in children and adolescents with diabetes to range from $1-10 \% \cdot{ }^{[4,5]}$ It is also in keeping with the study done in adult type 1 diabetic patients in Durban, SA, which showed a prevalence of biopsy-confirmed CD of $2.5 \% \cdot{ }^{[18]}$ Our prevalence seems to be lower than those reported in children in some other African countries, with a prevalence of $16.4 \%$ and $6.4 \%$ in Algeria and Egypt, respectively, while similar to the prevalence of $2.3 \%$ reported in Tunisia. ${ }^{[14-16]}$ More research in paediatric local prevalence rates of biopsy-confirmed CD would be valuable.

The present study found a higher predominance of coeliac serology positivity (82\%) as well as confirmed CD (100\%) in females compared with males; this is in keeping with international literature as well as local studies. ${ }^{[17]}$ In patients who had positive coeliac screens, $73 \%$ were asymptomatic, which is in keeping with a systematic review in paediatric patients in which $85 \%$ of patients were asymptomatic at diagnosis. ${ }^{[22]}$ This further emphasises the need for routine screening of all type 1 diabetic children as per international guidelines in order to minimise long-term morbidity and possibly mortality. ${ }^{[4,5]}$ Interestingly, none of the patients tested in this study was found to have an IgA deficiency. Irrespectively, all patients underwent both IgA and IgG serological testing, thus eliminating false negative coeliac antibodies.

Sixty-five percent of the patients screened were black; however, this reflects our local population. It is also important to note that higher-level socioeconomic children from the private sector were not included. The effect of ethnicity on the prevalence rate of $\mathrm{CD}$ remains unclear; however, weak evidence has suggested that it is rare in black patients. ${ }^{[18]}$ Our study shows an equal prevalence of biopsy-confirmed CD in white and black patients. This is statistically not significant and cannot be interpreted effectively owing to the small sample size and the predominance of black patients in this study; however, it does suggest that CD may very well not be rare in black patients.

Recent ISPAD guidelines recommend that symptomatic children with high tTG-A titres ( $\geq 10 \mathrm{x}$ ULN) may be diagnosed with $\mathrm{CD}$ without a small-bowel biopsy, but only if the EMA is also positive or if the patient carries HLA-DQ2 or HLA-DQ8. ${ }^{[4,5]}$ This recommendation is inconsistent with some other guidelines, but is consistent with recent guidelines from ESPGHAN.$^{[3,4,20]}$ ESPGHAN guidelines also state that HLA testing is not an obligatory criterion for a serology-based diagnosis of CD without biopsy (Fig. 2). ${ }^{[20]}$ If we had used this approach in our study, both patients confirmed to have biopsy-proven CD could have been diagnosed with definite CD without a biopsy, purely based on serology testing $\geq 10 \mathrm{x}$ ULN, using anti-DPG as the second sample. The use of anti-DPG as the second sample in place of EMA is, however, not documented in international guidelines and further research in this regard would be valuable for centres in which EMA is not available for testing. Conclusions in this regard cannot be made owing to the small sample size.

It is not surprising that almost all the serology-positive patients who did not have biopsy-confirmed $\mathrm{CD}$ were shown to still have abnormal intestinal biopsies (two (18\%) patients were, however, not biopsied). Several studies of intestinal integrity in patients with type 1 diabetes have shown evidence of increased intestinal permeability. ${ }^{[23,24]}$ The intestinal microbiome contributes a great deal to the maintenance of intestinal integrity. Type 1 diabetics tend to have bacteria in their gut microbiomes that have increased expression of genes related to adhesion and motility compared with controls, with some studies showing an increase in Bacteriodetes (associated with beta cell autoimmunity in children). ${ }^{[23-25]}$ Microstructural changes, including changes to tight junctions and microvilli, are frequently seen in the intestines of patients with type 1 diabetes. Intestinal biopsies in these patients have revealed higher densities of interleukin $1 \alpha$ and interleukin 4 cells, which suggest a heightened intestinal inflammatory state in type 1 diabetics. ${ }^{[23]}$ Small-bowel biopsies of type 1 diabetics exposed to gliadin reveal an exaggerated inflammatory response and thus, gliadin exposure has been shown to further affect intestinal integrity in these patients. ${ }^{[23]}$

Our study further supports the concept of gut dysbiosis with a heightened inflammatory state, as the remaining abnormal intestinal biopsies showed a picture of chronic gastritis and chronic duodenitis. Two biopsies also cultured $H$. pylori infection, with one patient also having a co-existing Giardia infection. A further study in a mixed paediatric and adult cohort of 240 patients with biopsy-proven $\mathrm{CD}$ found peptic lesions in the stomach or duodenum in $12 \%$ on endoscopy; however, no control group was reported and, in another retrospective study, abnormal findings were reported in 11 out of 115 paediatric patients. ${ }^{[20]}$ Premature conclusions regarding gut dysbiosis cannot be deduced from this study owing to the minimal number of patients requiring intestinal biopsy. It would be difficult to ethically justify intestinal biopsies for all the type 1 paediatric patients to evaluate gut dysbiosis and inflammatory states in asymptomatic children without proven benefit or intervention in this regard.

Our study had some limitations. It was predominantly a retrospective study and, as a result, some patients had insufficient serological testing. The capturing of signs and symptoms also 


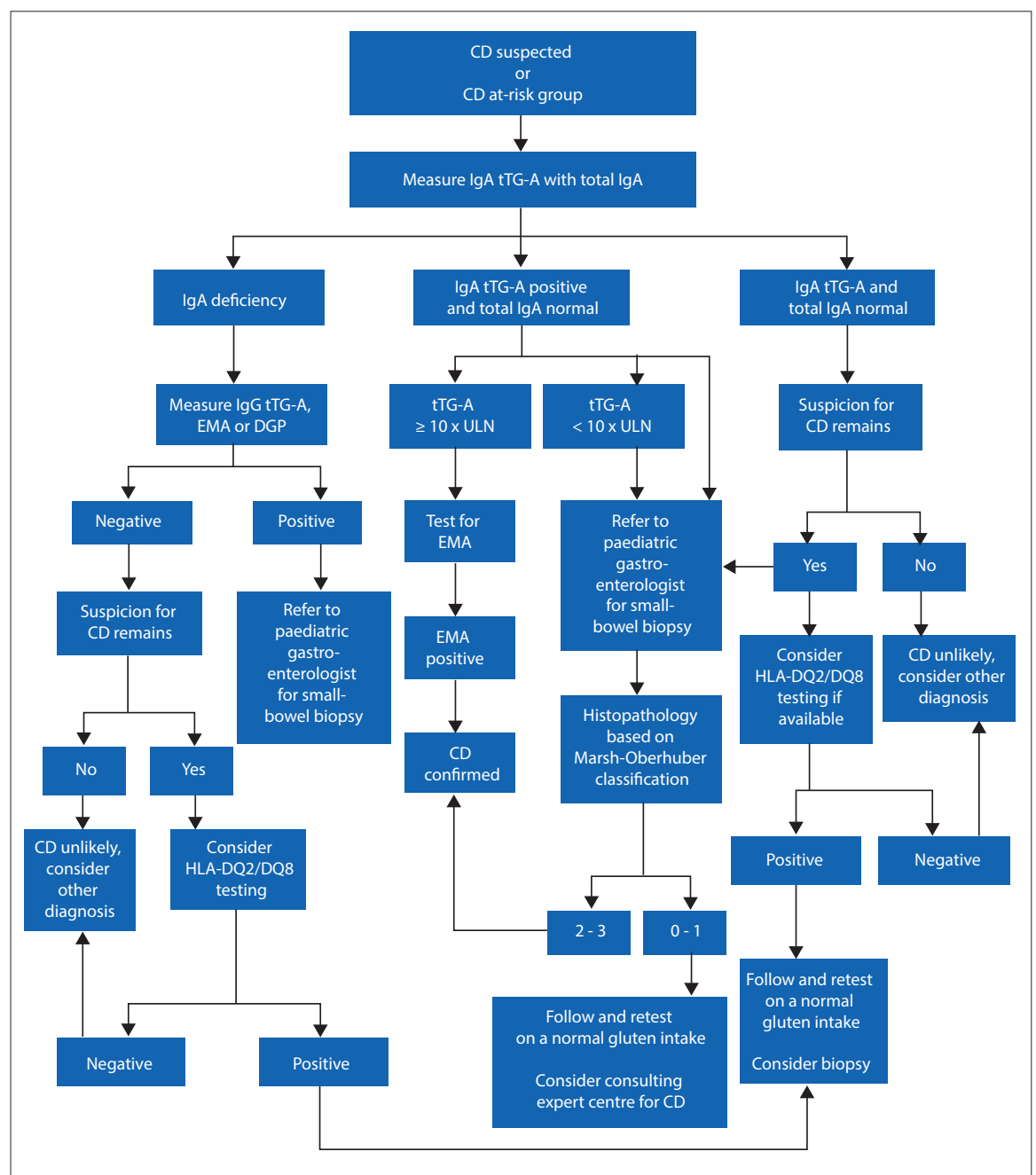

Fig. 2. Approach to coeliac disease (CD) diagnosis (adapted from Admou et al. and Pelkowski and Viera). ${ }^{[1,3]}$

relied on retrospective chart reviews. Two patients requiring gastrointestinal biopsies also relocated, were lost to follow-up and we were unable to contact them. This study is also limited by a lack of control subjects, the lack of inclusion of children from the private sector (with different dietary exposure) and the inability of our laboratory to test for EMA. HLA analysis was unfortunately also not performed owing to resource limitations. Only a small number of patients required biopsy, as it was not ethically justifiable to expose patients to unnecessary procedures; however, all our biopsies were standardised in the way in which they were taken by one of two specialists.

This study was not specifically designed to establish whether screening of asymptomatic children (even if they are at risk) is valuable, in general and in the South African context. Some literature has questioned the riskbenefit ratio of screening asymptomatic children for $\mathrm{CD}^{[26,27]}$ This evidence is, however, for all children and not specifically for at-risk groups, namely diabetic children who are mostly asymptomatic. ${ }^{[26,27]}$ The prevalence of CD in our South African population of type 1 diabetic children correlates with international prevalences and therefore it would be worthwhile following the ISPAD and ESPAGN guidelines, as this would not only aid in the earlier diagnosis and treatment of CD in these children but would also aid in the management of their concurrent diabetes mellitus.

\section{Conclusion}

This study found a prevalence of serologypositive CD in our local population of South African children with type 1 diabetes mellitus of $10.2 \%$, while the prevalence of biopsyconfirmed CD was found to be $1.9 \%$. This finding is in keeping with international literature, which has shown the prevalence of $\mathrm{CD}$ in children and adolescents with diabetes to range from $1-10 \%$. Therefore, international guidelines for screening of these patients are applicable to our patients and should be followed as far as possible, taking into account local resources. More research from other sub-Saharan countries, especially in paediatric patients, is required to verify the findings of this study and assist in the formation of local South African guidelines.

Declaration. Ethical approval for this study was obtained from the Research Ethics Department of the University of Pretoria (ref. no. 695/2018) and the National Health Research Database. All research was conducted according to the principles outlined in the Declaration of Helsinki. Acknowledgements. We thank Dr Ernst Muller of the Department of Paediatric Surgery, University of Pretoria, and Professor Piet Becker of the Department of Statistics, University of Pretoria, for their invaluable inputs.

Author contributions. The authors confirm contributions to this paper as follows: study conception and design by MK, AJT, TK, JCvD; data collection, analysis and interpretation of results by MK; draft manuscript preparation by MK, AJT, TK, JCvD. All authors reviewed the results and approved the final version of the manuscript.

Funding. None.

Conflicts of interest. None.

1. Admou B, Essaadouni L, Krati K, et al. Atypical celiac disease: From recognizing to managing Gastroenterol Res Pract 2012;637187:1-9. https:// doi.org/10.1155/2012/637187

2. Freemark M, Levitsky LL. Screening for celiac disease in children with type 1 diabetes. Diabetes Care 2003;26(6):1932-1939. https://doi. org/10.2337/diacare.26.6.1932

3. Pelkowski TD, Viera AJ. Celiac disease diagnosis and management. Am Fam Physician 2014;89(2):99-105.

4. Kordonouri O, Klingensmith G, Knip M, et al. ISPAD Clinical Practice Consensus Guidelines 2014 Compendium. Other complications and diabetesassociated conditions in children and adolescents. Pediatric Diabetes 2014;15(Suppl.20):270-278. https://doi.org/10.1111/pedi.12183

5. Mahmud FH, Elbarbary NS, Fröhlich-Reiterer E, et al. ISPAD Clinical Practice Consensus Guidelines 2018: Other complications and associated conditions in children and adolescents with type 1 diabetes. Paediatric Diabetes. 2018;19:275-286. https://doi.org/10.1111/pedi.12740

6. Yildirmaz S, Altay D, Esen I, et al. Prevalence of celiac disease in children with type 1 diabetes mellitus in southeast region of Turkey. In J Clin Pediatr 2016;5(2):32-35. https://doi. org/10.14740/ijcp239w

7. Hansen D, Brock-Jacobsen B, Lund E, et al. Clinical benefit of a gluten-free diet in type 1 diabetic children with screening-detected celiac disease. Diabetes Care 2006;29(11):2452-2457. https://doi.org/10.2337/dc06-0990

8. Goh C, Banerjee K. Prevalence of coeliac disease in children and adolescents with type 1 diabetes mellitus in a clinic based population. Postgrad Med J 2007;83:132-136. https://doi.org/10.1136/ pgmj.2006.049189

9. Craig ME, Prinz N, Boyle CT, et al. Prevalence of celiac disease in 52,721 youth with type 1 diabetes: international comparison across three continents. Diabetes Care 2017;40(8):1034-1040. https://doi.org/10.2337/dc16-2508 
10. Joshi R, Madvariya M. Prevalence and clinical profile of celiac disease in children with type 1 diabetes mellitus. Indian J Endocrinol Metab 2015;19(6):797-803. https://doi.org/10.4103/2230-8210.167555

11. Goncalves CBCD, Silva IN, Tanure MG, et al. Study of prevalence of celiac disease in children with type 1 diabetes mellitus: Result of 10 years of followup. Arq Bras Endocrinol Metab 2013;57(5):375-380. https://doi.org/10.1590/ s0004-27302013000500007

12. Al-Sinani S, Sharef SW, Al-Yaarubi S, et al. Prevalence of celiac disease in Omani children with type 1 diabetes mellitus: A cross sectional study. Oman Medical Journal 2013;28(4):260-263. https://doi.org/10.5001/omj.2013.73

13. Alghamdi AH, Nasaif MN, Dammas AS, et al. Trends of diabetes in children and adolescents in pediatric endocrinology clinic and clinical pediatric diabetes centre at Al Baha Central Hospital in Saudi Arabia. JPMC 2016;1(3):921. https://doi.org/10.14302/issn.2474-3585.jpmc-16-1281

14. Moayeri H, Bahremand SH. Prevalence of celiac disease in children and adolescents with type 1 diabetes mellitus. Medical J Islamic Republic of Iran 2004;18(1):39-43

15. Zamanfar D, Aarabi M, Sadeghian I. Prevalence of celiac disease in children with type 1 diabetes mellitus: A review of literatures in the Islamic Republic of Iran. J Pediatr Rev 2014;2(1):10-16.

16. Bouguerra R, Ben Salem L, Chaâbouni H, et al. Celiac disease in adult patients with type 1 diabetes mellitus in Tunisia. Diabetes Metab 2005;31:83-86. https:// doi.org/10.1016/s1262-3636(07)70171-4

17. Tayob S, Pillay K, Tlou B, et al. Prevalence of positive coeliac serology in a cohort of South African children with type 1 diabetes mellitus. S Afr J Child Health 2016;10(1):12-15. https://doi.org/10.7196/sajch.2016.v10i1.835

18. Paruk IM, Naidoo VG, Pirie FJ, et al. Prevalance and characteristics of celiac disease in South African patients with type 1 diabetes mellitus: Results from the Durban Diabetes and Celiac Disease Study. J Gastroenterol Hepatol 2019;34:673-678. https://doi.org/10.1111/jgh.14596
19. Stanford Medicine. Surgical Pathology Criteria. http://surgpathcriteria.stanford. edu/gi/celiac-disease/marsh.html (accessed 29 July 2018).

20. Husby S, Koletzko S, Korponay-Szabó I, et al. European Society Paediatric Gastroenterology, Hepatology and Nutrition Guidelines for Diagnosing Coeliac Disease 2020. J Pediatr Gastroenterol Nutr 2020;70(1):141-157. https://doi org/10.1097/mpg.0000000000002497

21. Liu E, Bao F, Barriga $\mathrm{K}$, et al. Fluctuating transglutaminase autoantibodies are related to histologic features of celiac disease. Clin Gastroenterol Hepatol 2003;1:356-362. https://doi.org/10.1053/s1542-3565(03)00180-0

22. Pham-Short A, Donaghue KC, Ambler G, et al. Screening for celiac disease in type 1 diabetes: A systematic review. Paediatrics 2015;136(1):171-176. https:// doi.org/10.1542/peds.2014-2883

23. Boerner BP, Sarvetnick NE. Type 1 diabetes: Role of intestinal microbiome in humans and mice. Ann NY Acad Sci 2011;1243:103-118. https://doi.org/10.1111/ j.1749-6632.2011.06340.x

24. Zheng P, Li Z, Zhou Z. Gut microbiome in type 1 diabetes: A comprehensive review. Diabetes Metab Res Rev 2018;34:1-9. https://doi.org/10.1002/dmrr.3043

25. Cohn A, Sofia MA, Kupfer SS. Type 1 Diabetes and celiac disease: Clinical overlap and new insights into disease pathogenesis. Curr Diab Rep 2014;14(8):116. https://doi.org/10.1007/s11892-014-0517-x

26. Choung RS, Murray JA. The US Preventive Services Task Force recommendation on screening for asymptomatic celiac disease: A dearth of evidence. JAMA 2017;317(12):1221-1223. https://doi.org/10.1001/jama.2017.1105

27. Chou R, Bougatsos C, Blazina I, et al. Screening for celiac disease: Evidence report and systematic review for the US Preventive Services Task Force. JAMA 2017;317(12):1258-1268. https://doi.org/10.1001/jama.2016.10395

Accepted 13 January 2021. 